\title{
NEUMOPERITONEO ASINTOMÁTICO ASOCIADO A NEUMATOSIS QUÍSTICA INTESTINAL EN PACIENTE QUIRÚRGICO*
}

\author{
Drs. María Marqueta S. ${ }^{1}$, Jimena Cubero C. ${ }^{2}$, Lorena Rodríguez G. ${ }^{1}$, \\ Begoña Corral R. ${ }^{2}$, Norberto Herrera M. ${ }^{1}$, Juan Manuel Fernández G. ${ }^{2}$ \\ 1 Servicio de Cirugía General y Aparato Digestivo. Hospital Severo Ochoa. Leganés. \\ 2 Servicio de Radiodiagnóstico. Hospital Severo Ochoa. Leganés. \\ Madrid, España.
}

\begin{abstract}
Asymptomatic pneumoperitoneum associated to pneumatosis cystoides intestinalis in surgery patient
\end{abstract}

Introduction: Pneumatosis cystoides intestinalis (PCI) is a rare entity characterized by the presence of air in the intestinal wall. In most cases, it is secondary to a number of mainly intra-abdominal processes that mostly require urgent surgery but, in a small percentage of cases, it is idiopathic and usually has a more benign course, caused by, among other things, a series of mechanical factors causing mucosal damage and therefore predisposing to the formation of cysts. Case report: Here we describe a case of a patient undergoing right hemicolectomy for colon cancer and subsequently treated with chemotherapy that developed PCI two years after treatment ended. At all times, the patient was asymptomatic, despite the evolution of PCI and the development of associated pneumoperitoneum.

Key words: Pneumatosis cystoides intestinalis, surgery, chemotherapy, pneumoperitoneum.

\section{Resumen}

Introducción: La neumatosis quística intestinal (NQI) es una entidad poco frecuente que se caracteriza por la presencia de aire en la pared intestinal. En la mayoría de las ocasiones es secundaria a una serie de procesos fundamentalmente intrabdominales y que en su mayoría requieren cirugía urgente, pero en un pequeño porcentaje de casos es idiopática y suele tener un curso más benigno, causada entre otras cosas por una serie de factores mecánicos que ocasionan el daño de la mucosa y por lo tanto predisponen a la formación de quistes. Caso clínico: Presentamos el caso de un paciente sometido a una hemicolectomía derecha por cáncer

*Recibido el 15 de junio de 2015 y aceptado para publicación el 23 de agosto de 2015.

Los autores declaran no tener conflicto de intereses.

Correspondencia: Dra. María Marqueta S. mmarqueta@hotmail.com 
de colon y tratado posteriormente con quimioterapia que desarrolla una NQI a los dos años de finalizado el tratamiento. En todo momento el paciente se ha encontrado asintomático a pesar de la evolución de la NQI y el desarrollo de neumoperitoneo asociado.

Palabras clave: Neumatosis quística intestinal, cirugía, quimioterapia, neumoperitoneo espontáneo.

\section{Introducción}

La NQI se caracteriza por la presencia de aire en la pared intestinal. La mayoría de las veces el aire se queda atrapado en la submucosa, llegando a alcanzar la subserosa en raras ocasiones sobre todo en los casos de neumatosis de intestino delgado. Suele aparecer asociada a diversos procesos intraabdominales, pero en un $15 \%$ de los casos se habla de NQI primaria o idiopática en cuya etiología intervienen diversos factores.

En general suele cursar de forma asintomática y la exploración abdominal suele ser anodina. Los síntomas más frecuentes en los casos de afectación del intestino grueso son la diarrea, hematoquecia y dolor abdominal ${ }^{1}$.

Descartando las causas de neumatosis intestinal provocadas por procesos abdominales que se manifiestan con abdomen agudo, las causas más frecuentes de NQI son las siguientes: procesos pulmonares, infecciosos, enfermedades del tubo digestivo que afecten a la mucosa intestinal y a la motilidad, traumatismos como procesos endoscópicos y quirúrgicos y la inmunosupresión. En su diagnóstico se pueden emplear varios métodos de imagen, pero quizá el más rentable sea la TC, sobre todo en los casos sintomáticos para tratar de filiar la causa. En los casos de NQI primaria no suele ser necesario aplicar ningún tratamiento, y la mayoría de las veces se resuelven de manera espontánea. Para pacientes poco sintomáticos el tratamiento se basa en la dieta y antibioterapia con metronidazol, y para aquellos más sintomáticos está indicado añadir al tratamiento anterior la oxigenoterapia.

Describimos un caso de un paciente al que se le realizó una hemicolectomía derecha laparoscópica por un cáncer de colon, que en el seguimiento posterior a los dos años de la cirugía, aparece como hallazgo casual en una prueba de imagen una neumatosis intestinal que cursa de forma asintomática.

\section{Caso clínico}

Varón de 74 años de edad con antecedentes personales de HTA, poliartrosis, oftalmoplejia dolorosa recidivante sin secuelas, paludismo en la infancia e intervenido quirúrgicamente de herniorrafia inguinal, apendicectomía y timpanoplastía derecha, que presenta en marzo de 2010 una neoplasia de colon derecho a nivel del ángulo hepático. En el estudio preoperatorio los marcadores tumorales son normales y el TC abdominal no muestra enfermedad a distancia. En marzo de 2010 se realiza una hemicolectomía derecha laparoscópica sin complicaciones en el postoperatorio salvo rectorragia autolimitada sin repercusión hemodinámica. El diagnóstico final fue de adenocarcinoma de colon derecho estadio IIIA.

Valorado por el Servicio de Oncología recibe ocho ciclos de quimioterapia, los dos primeros con una combinación de oxaliplatino y capecitabina y los seis últimos sólo con capecitabina por toxicidad al oxaliplatino. En abril de 2012 se realiza un TC abdominal informado como presencia de burbujas
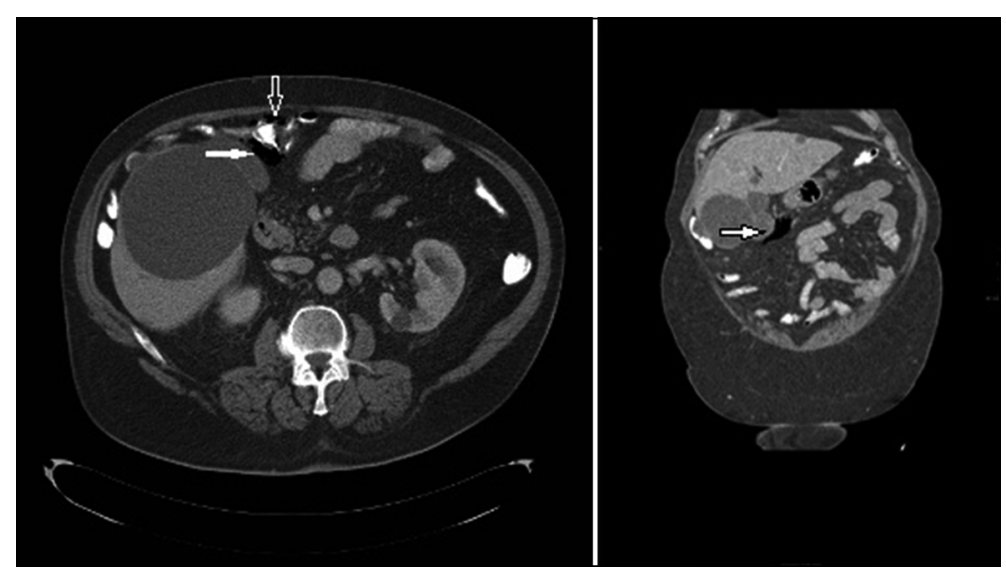

Figura 1. Estudio de TC abdomen con contraste oral e intravenoso (abril de 2012). Control rutinario en paciente asintomático con hemicolectomía derecha laparoscópica que recibió tratamiento con quimioterapia. En la imagen axial se identifica la presencia de burbujas aéreas intramurales (flecha hueca) e intraperitoneales (flecha sólida) adyacentes a la sutura quirúrgica. En la reconstrucción coronal se visualiza la salida de gas a cavidad abdominal (flecha sólida). No se evidencia fuga de contraste. 

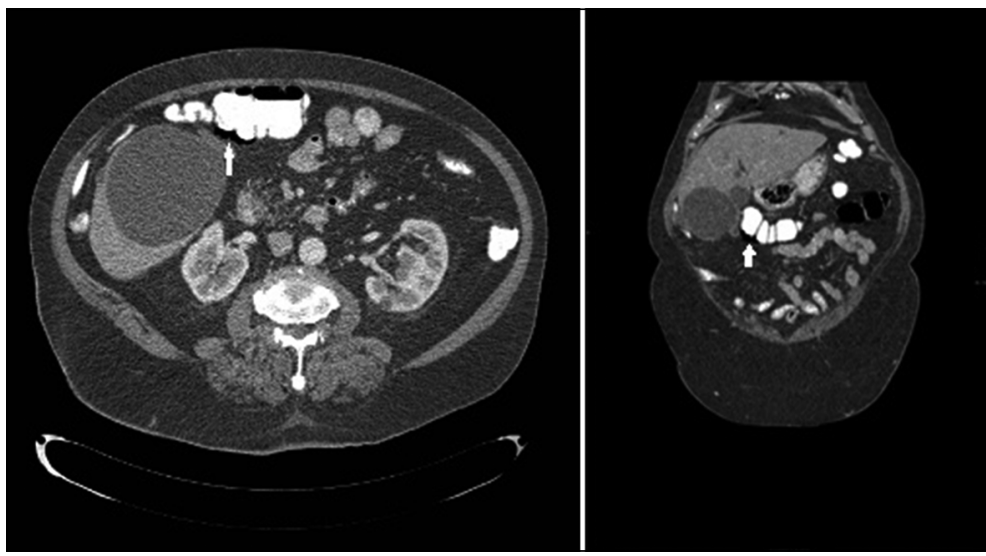

Figura 2. Estudio de TC abdomen con contraste oral, intravenoso y enema de gastrografía (junio de 2012) del mismo paciente. El estudio axial y las reconstrucciones coronales muestran la persistencia del neumoperitoneo que ha disminuido con respecto a estudio previo (flechas sólidas).

aéreas adyacentes a la sutura quirúrgica compatible con el diagnóstico de neumatosis intestinal. Ante los hallazgos en las pruebas de imagen se realiza un nuevo TC con contraste oral y rectal en junio de 2012, siendo informado como neumatosis intestinal en colon transverso adyacente a la sutura quirúrgica con salida de aire a la cavidad abdominal adyacente a la sutura y el hipocondrio izquierdo, que han aumentado de tamaño con respecto al estudio previo, no se objetiva fuga de contraste. Posteriormente se realiza una colonoscopia en julio de 2012 (tenía una previa en 2011, antes del diagnóstico de NQI, informada como normal) en la que se observa la anastomosis T-L de aspecto normal y resto sin alteraciones.

El paciente en todo momento ha estado asintomático a pesar de aumentar tanto la neumatosis intestinal como la cantidad de aire ectópico intraabdominal y no ha mostrado progresión de la enfermedad hasta el momento actual. En ningún momento ha recibido tratamiento.

\section{Discusión}

La NQI es una entidad poco frecuente que se caracteriza por la presencia de aire en la pared intestinal. Fue descrita por primera vez por Bang en 1876. El 85\% de las veces es secundaria a diversos procesos tanto intraabdominales como extraabdominales que en la mayoría de los casos conducen a la necrosis intestinal y a la posterior perforación. Estos casos suelen requerir una intervención quirúrgica urgente. El 15\% restante se considera primaria o idiopática en cuya etiología intervienen varios factores. Generalmente suele tener un curso más benigno y es raro que requiera una cirugía urgente.

En su etiopatogenia están implicadas diversas teorías ${ }^{2}$ : la mecánica que se basa en el daño de la mucosa como factor predisponente para la salida de aire hacia la pared intestinal; la bacteriana que hace referencia a la producción de gas por bacterias como el clostridium perfringens hacia la luz intestinal que provoca la salida del mismo hacia la pared; una teoría bioquímica que habla del cambio de composición de los gases intestinales aumentando la concentración de hidrógeno, esto se ve favorecido por la toma de inhibidores de la $\alpha$ glucosidasa; y una teoría pulmonar en la cual se hace referencia al paso de aire desde el árbol bronquial hasta los vasos mesentéricos y finalmente a la pared intestinal.

El desarrollo de neumatosis intestinal en nuestro caso parece estar relacionado con factores mecánicos. Tanto la cirugía ${ }^{3}$ como los quimioterápicos provocan un daño en la mucosa que parece ser el causante, entre otros factores del desarrollo de la NQI. Pero lo sorprendente de este caso es que la neumatosis intestinal aparezca como hallazgo casual dos años después de la cirugía, y varios meses después de terminar con los ciclos de quimioterapia.

Revisando la literatura, hemos encontrado una descripción similar de tres casos en los que la NQI aparece dos años después de la intervención quirúrgica, también en el colon derecho y también en relación con la anastomosis quirúrgica y la quimioterapia, si bien en estos casos todos los pacientes presentaban títulos de ANA positivos con una posible relación con enfermedades del colágeno, mientras que en nuestro paciente estos títulos son negativos. Otro dato diferencial es la presencia de neumoperitoneo, en general suele ser sinónimo de mala evolución y suele asociar la realización de una laparotomía exploradora, pero en este caso el paciente permanece asintomático.

En la bibliografía revisada, la neumatosis intestinal en el colon suele caracterizarse por la presencia 
de quistes en la submucosa, mientras que en nuestro caso se localizan en la subserosa. Por otro lado, se han realizado dos colonoscopias separadas con un intervalo de un año en las cuales no se ha observado ningún signo de neumatosis intestinal, algo lógico teniendo en cuenta que los quistes en nuestro paciente son subserosos. Esto acompañado del hecho de que el aire en la pared haya aumentado con el tiempo en lugar de disminuir, puede explicar la presencia de neumoperitoneo debido a la hiperpresión que provoca el aire ectópico en la pared intestinal. Al estar en la subserosa sería más fácil su ruptura y su consiguiente relación con el neumoperitoneo.

Hay varios factores en nuestro caso que coinciden con lo ya publicado, lo que lo diferencia del resto es la secuencia temporal. Tanto la cirugía, como la quimioterapia, como incluso la realización de colonoscopias de control se han asociado en mayor o menor medida con esta entidad, si bien es cierto que por lo general suele haber una relación causa-efecto más inmediata.

\section{Referencias}

1. Jamart J. Pneumatosis Cystoides Intestinalis, a stadistical study of 919 cases. Acta Hepato-Gastroenterol. 1979;26:419-22.

2. Selles-Dechent R, Zumarraga-Navas P, Ruiz del Castillo J. Neumoperitoneo debido a neumatosis quística intestinal. Cir Esp. 2004;76:396-9.

3. Wandtke J, Skucas J, Spataro R, Bruneau RJ. Pneumatosis intestinales as a complication of Jejunoileal Bypass. A J Roentgenol. 1977;129:601-4.

4. Mimatsu K, Oida T, Kawasaki A, Kano H, Kuboi Y, Aramaki O, Armano S. Pneumatosis cystoides intesti- nales alter fluorouracil chemotherapy for rectal cancer. World Journal of Gastroenterology 2008;14:3273-5.

5. Horiuchi A, Akamatsu T, Mukawa K, Ochi Y, Arakura N, Kiyosawa K. Pneumatosis cystoides intestinales associated with post-surgical anastomosis: a report of three cases and review of the Japanese literature. Journal of Gastroenterology and Hepatology 1998;13:534-7.

6. Boerner M, Fried DB, Warshauer DM, Isaacs K. Pneumatosis intestinalis, two case reports and a retrospective review of the literature from 1985 to 1995 . Digestive Diseases and Sciences 1996;41:2272-85.

7. López Cano M, Vilallonga Puy R, Lozoya Trujillo R, Espin Basany E, Sánchez García J, Armengol Carrasco M. Neumoperitoneo idiopático. Cir Esp. 2005;78:112-4.

8. Menchén BJ, Alcalde F, Campano I, Rodríguez E, Morales C, Sánchez Bustos F, y cols. Una causa rara de neumoperitoneo espontáneo: neumatosis quística. Cir Esp. 2002;72:306.

9. Azzaroli F, Turco L, Sartoni Galloni S, Buonfiglioli F, Calvanese C, Mazzella G. Pneumatosis cystoides intestinalis. World Jounal of Gastroenterology 2011;17:49326.

10. Arikanoglu Z, Aygen E, Camci C, Basbug M, Dogru O, Cetinkaya Z, et al. Pneumatosis cystoides intestinalis: a single center experience. World Journal of Gastroenterology 2012;18:453-7.

11. Ivanovic A, Kovac J, Masulovic D, Stefanovic A, Jaksic E, Saranovic D. The role of multidetector computer tomography in diagnosis of neumatosis cystoides intestinalis. Journal of Gastroenterology and Hepatology 2012;27:182.

12. St Peter SD, Abbas MA, Kelly KA. The spectrum of pneumatosis intestinalis. Arch Surg. 2003;138:68-75.

13. Shapiro BJ, Track AA, Myers E. Pneumatosis cystoides intestinalis involving the left side of the colon. Canad Med Ass J. 1964;91:219-24. 